\title{
The effect of borate bioactive glass on the printability of methylcellulose-manuka honey hydrogels
}

\author{
Katharina Schuhladen ${ }^{1, b)}$, Vera Bednarzig ${ }^{1, b)}$, Nadine Rembold ${ }^{1}$, Aldo R. Boccaccini ${ }^{1, a)}$ \\ ${ }^{1}$ Department of Materials Science and Engineering, Institute of Biomaterials, University of Erlangen-Nuremberg, 91058 Erlangen, Germany \\ a) Address all correspondence to this author. e-mail: aldo.boccaccini@ww.uni-erlangen.de \\ b) These two authors share first co-authorship. \\ Received: 25 February 2021; accepted: 19 May 2021; published online: 8 June 2021
}

\begin{abstract}
Aldo R. Boccaccini was a guest editor of this journal during the review and decision stage. For the JMR policy on review and publication of manuscripts authored by editors, please refer to http://www.mrs.org/editor-manuscripts/.

3D printing offers the possibility to generate complex and individualized constructs (scaffolds) for applications in tissue engineering. This is viable by using suitable inks based on advanced biomaterials. Methylcellulose (MC), a highly biocompatible biomaterial, can be combined with manuka honey (H) to fabricate a thermo-sensitive hydrogel. Besides providing favorable biological effects, $\mathrm{H}$ can also be used as a natural cross-linking agent. Furthermore, the addition of bioactive glass (BG) to the ink could improve its mechanical and bioactive properties. In this study, a composite based on MC as matrix incorporating $\mathrm{H}$ and particulate borate $\mathrm{BG}$ as filler, was investigated as ink for 3D printing. Besides the improvement of the inks' printability owing to the addition of BG, the printed scaffolds exhibited suitable swelling behavior and mechanical properties. Moreover, cell biology tests demonstrated the potential of the composite for biofabrication and applications in tissue engineering, which should be further explored.
\end{abstract}

\section{Introduction}

Based on its capability for fabricating patient-specific implants and scaffolds in different sizes and shapes and with required complexity in short time, 3D printing has gained attention as a leading technique in the field of tissue engineering and regenerative medicine. The main component of $3 \mathrm{D}$ printing, the ink, has to fulfill a list of requirements, such as being printable, biodegradable, and offering suitable mechanical properties adjusted to the intended field of application $[1,2]$. The thermo-sensitive polymer methylcellulose (MC) is a very attractive material for 3D printing based on its reversible sol-gel transition behavior [3]. MC is a water soluble cellulose derivative, where methyl groups substitute hydroxyl groups [4]. Below the so-called critical solution temperature, water molecules surround the hydrophobic methyl groups, which makes MC hydrophilic and lipophilic at the same time $[5,6]$. Above the critical solution temperature, water molecules do not surround the methyl groups anymore, leading to the exposure of the hydrophobic groups [5]. The presence of these hydrophobic groups leads to an increase of viscosity and, as a result of this, to the formation of a gel [4]. Interestingly, the temperature at which the sol-gel transition occurs can be modified by the addition of a salt during the hydrogel fabrication [6,7]. Among other advantages, $\mathrm{MC}$ is a highly biocompatible material, which is approved by the US Food and Drug Administration (FDA) for several applications, e.g., for use in pharmaceuticals [8]. As we showed in previous studies $[9,10]$, MC can be chemically crosslinked with manuka honey $(\mathrm{H})$, by the action of methylglyoxal groups present in the honey, which has been proven to cross-link cellulose [11]. Among its other favorable biological effects in wound healing (e.g., antibacterial effect, stimulation of wound epithelialization and anti-inflammatory efficiency), $\mathrm{H}$ is an interesting natural cross-linker, which makes unnecessary the addition of other standard and potentially cytotoxic cross-linkers, such as glutaraldehyde, which could have negative effects on the biological system [12-15].

By introducing bioactive glass (BG) particles in $\mathrm{MC}-\mathrm{H}$ hydrogels, a dual biological effect of the manuka honey and the 
released ions from the BG particles can be achieved [16]. BG is a well-known bioactive inorganic material (for example 45S5 BG of composition (in wt\%): $45 \mathrm{SiO}_{2}-24.5 \mathrm{CaO}-24.5 \mathrm{Na}_{2} \mathrm{O}-6 \mathrm{P}_{2} \mathrm{O}_{5}$ ) due to its dissolution ability in aqueous solutions, which induces the release of biologically active ions for specific cell responses $[17,18]$. Besides providing favorable biological activities (such as angiogenic, osteogenic and antibacterial effects), the addition of BG particles to biopolymers can lead to an improvement of the mechanical properties of the composites $[19,20]$. Originally, BGs have mainly been considered for bone tissue engineering and orthopedic applications [18]; however, more recently, the efficient use of BGs, including borate glasses, in contact with soft tissues and for wound healing has been documented [21, 22].

In the framework of this study, we aim to evaluate for the first time the suitability of $\mathrm{MC}-\mathrm{H}$ based inks containing borate BG (micronsized) particles for their use in 3D printing. After the successful printing of scaffolds, the mechanical, swelling and chemical properties were evaluated. In addition, preliminarily cell biology tests using human dermal fibroblasts seeded on top of the printed structures were conducted as a preliminary assessment of the scaffolds' biological activity.

\section{Results}

As demonstrated in Fig. 1, 3D printing of MC-H inks was possible with and without BG addition. However, neat MC scaffolds were seen to collapse after a certain time period, these samples were therefore not further investigated and just used as a reference. In the FTIR spectra of all scaffolds (Fig. 2), the characteristic bands of $\mathrm{MC}$ can be found: the stretching vibrations of the $\mathrm{C}-\mathrm{O}$ oxygen bridge $\left(1150 \mathrm{~cm}^{-1}\right)$, the $\mathrm{C}-\mathrm{O}-\mathrm{C}$ stretching mode of the glycosidic unit $\left(1050 \mathrm{~cm}^{-1}\right)$ as well as the vibration of $\mathrm{OCH}_{3}$ groups $\left(940 \mathrm{~cm}^{-1}\right)$ [23]. Moreover, peaks related to sugar (peaks in the range of $750 \mathrm{~cm}^{-1}$ to $1500 \mathrm{~cm}^{-1}$ ), which is the main

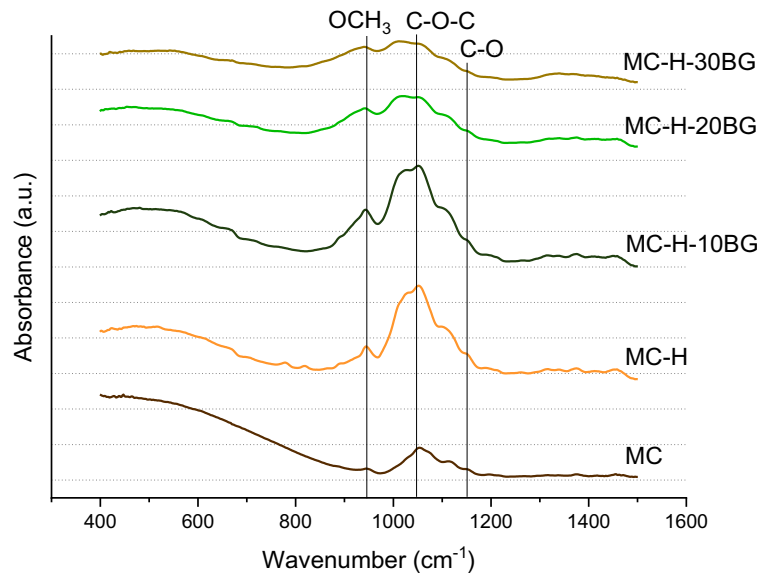

Figure 2: FTIR spectra of the MC-H-based scaffolds. Samples contain borate BG in different concentrations. Pure $M C$ is used as a basis for comparison.

component of manuka honey [14,24], can be observed in the spectra of honey containing scaffolds. However, the presence of BG inside the BG-containing samples cannot be clearly detected by FTIR. This effect was already reported previously, when BG of the same composition was used in the fields of electrospinning and freeze drying $[9,10]$. A reason for this behavior could be the immersion of the BG particles in the hydrogel matrix and the resulting surface coverage, which leads to no clearly detectable BG peaks inside the composite system. Nevertheless, the decrease of peaks related to $\mathrm{MC}$ and $\mathrm{H}$, especially in the absorbance spectrum from 900 to $1100 \mathrm{~cm}^{-1}$, could be considered an indirect indication of the presence of $\mathrm{BG}$. In this range, absorption bands related to the $\mathrm{B}-\mathrm{O}$ stretching of tetrahedral $\mathrm{BO}_{4}$ units in borate glasses [25] can be found (Fig. 2).

The mechanical properties of the printed MC-H based scaffolds were assessed by calculating the mean compressive strength in the range of $70-80 \%$ of the compressive strain, as
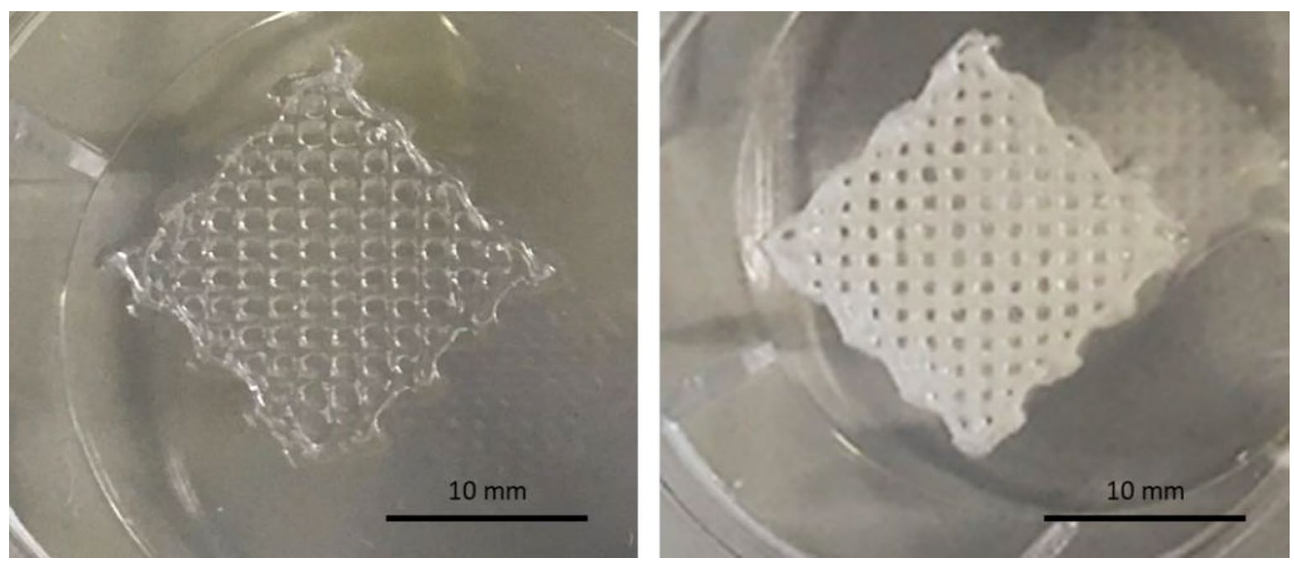

Figure 1: 3D-printed scaffolds (top view) of neat MC (left) and MC-H hydrogel with incorporated borate BG particles (right), with an edge length of $10 \mathrm{~mm}$ and a pore diameter of $0.9 \mathrm{~mm}$. 


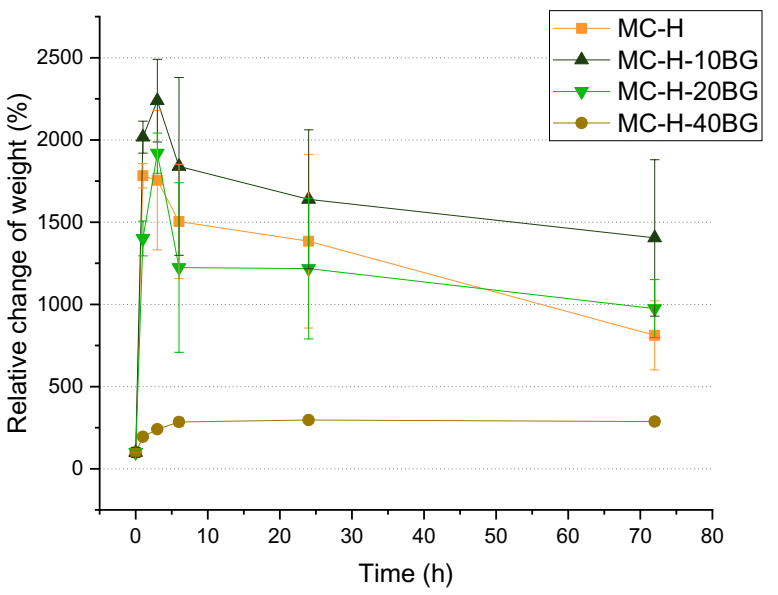

Figure 3: Relative change of weight (swelling capacity) of the MC-Hbased scaffolds containing different amounts of borate BG particles during immersion in DMEM for up to $72 \mathrm{~h}$.

TABLE 1: Summarized results of mechanical and contact angle tests of MC-H-based scaffolds containing different amounts of borate BG particles.

\begin{tabular}{lcc}
\hline \hline & $\begin{array}{l}\text { Mean compression strength } \\
\text { (70-80\% compressive strain) (kPa) }\end{array}$ & Contact angle \\
\hline MC-H & $5 \pm 1$ & $68 \pm 3^{\circ}$ \\
MC-H-10BG & $11 \pm 1$ & $76 \pm 14^{\circ}$ \\
MC-H-20BG & $7 \pm 1$ & $68 \pm 4^{\circ}$ \\
MC-H-40BG & $15 \pm 4$ & $35 \pm 8^{\circ}$ \\
\hline \hline
\end{tabular}

summarized in Table 1. Surprisingly, no significant difference between the mean compressive strength of $\mathrm{MC}-\mathrm{H}(5 \pm 1 \mathrm{kPa})$ and MC-H-20BG $(7 \pm 1 \mathrm{kPa})$ could be found, whereas the addition of 10 and $40 \mathrm{wt} \%$ of BG leads to a significant compressive strength improvement of up to $11 \pm 1 \mathrm{kPa}$ and $15 \pm 4 \mathrm{kPa}$, respectively.

In contrast to the mechanical properties, no significant influence resulting from the addition of borate BG particles on the wettability of the scaffolds was detected (Table 1). All MC-H based scaffolds exhibited a contact angle in the range $60^{\circ}-80^{\circ}$, indicating the successful incorporation of the $\mathrm{BG}$ particles in the polymer matrix. The only exception was found by the addition of $40 \mathrm{wt} \% \mathrm{BG}$ in the ink, which resulted in a significant reduction of the contact angle to a value of $35 \pm 8^{\circ}$.

To investigate the samples' swelling behavior, the printed scaffolds were immersed in DMEM for up to $72 \mathrm{~h}$. All scaffolds showed an initial fast water uptake (Fig. 3). With the exception of MC-H-40BG, all scaffolds exhibited a weight maximum after $3 \mathrm{~h}$ of immersion. This fast increase of weight in the initial hours followed by a slow release of water indicates the great capability of the scaffolds to absorb water. Interestingly, the addition of 10 $\mathrm{wt} \%$ of $\mathrm{BG}$ particles led to an increase of the swelling ratio in comparison to the neat MC-H-based scaffolds, whereas higher amounts of BG particles reduced the swelling capacity of the printed scaffolds. It should be mentioned that the reduction of weight with increasing immersion time might also be due to the fast dissolution of the borate BG particles, which has been already reported in literature [26], especially in samples containing $40 \mathrm{wt} \%$ of BG in the MC-H matrix. Since in these samples the highest concentration of glass is incorporated, the possible weight reduction effect of the BG particles should be most clearly detectable

To evaluate the biological potential of the developed ink and especially the effect of the incorporated BG particles for biofabrication, preliminary direct cell tests were performed using hDFs cells. Based on fluorescence images (Fig. 4) taken $24 \mathrm{~h}$ after the seeding of hDFs on top of the printed MC-H based scaffolds, no significant influence in comparison to the reference could be found. By measuring the relative viabilities of hDFs cultured on the different scaffolds 24 h post cell seeding using the WST- 8 assay, quantitative information can be obtained. As shown in Fig. 5, the results indicate no significant impact of the composition of the tested scaffolds on the viability of hDFs in comparison to the control (cells seeded on well plate). These results indicate the biocompatibility of the inks, which warrants their further investigation for application in the field of biofabrication.

\section{Discussion}

Although MC is a well-known material for the fabrication of cell sheets [6,7] or for the 3D printing of blends [27-29], there are only few studies reporting the suitability of neat $\mathrm{MC}$ as a biocompatible ink for 3D printing $[6,7]$. Based on the results obtained in previous studies [6,7], MC inks have been prepared by using PBS, a salt containing solution able to reduce the gel formation temperature of MC [30-32]. Even if it was possible to use the obtained neat MC ink for 3D printing, the scaffolds were not stable and collapsed after a few hours. One possible approach to improve the stability of the scaffolds would be to further increase the salt concentration during the preparation of the ink. However, this could also lead to undesirable side effects for applications in contact with cells (especially in the case of biofabrication) [33]. Therefore, this approach was not further investigated.

Based on previous results focusing on the use of $\mathrm{MC}-\mathrm{H}$ solutions for electrospinning and freeze drying $[9,10]$, the increase of the stability of MC scaffolds was investigated by considering the addition of $\mathrm{H}$, achieving a (mild) cross-linking effect. Indeed, the stability of the hydrogel was shown to be improved by adding $\mathrm{H}$ to $\mathrm{MC}$ in a ratio of 1:2. The obtained scaffolds were stable over a longer period (several days) in comparison to neat MC inks, although the printing speed had to be reduced, which led to a relatively long fabrication 

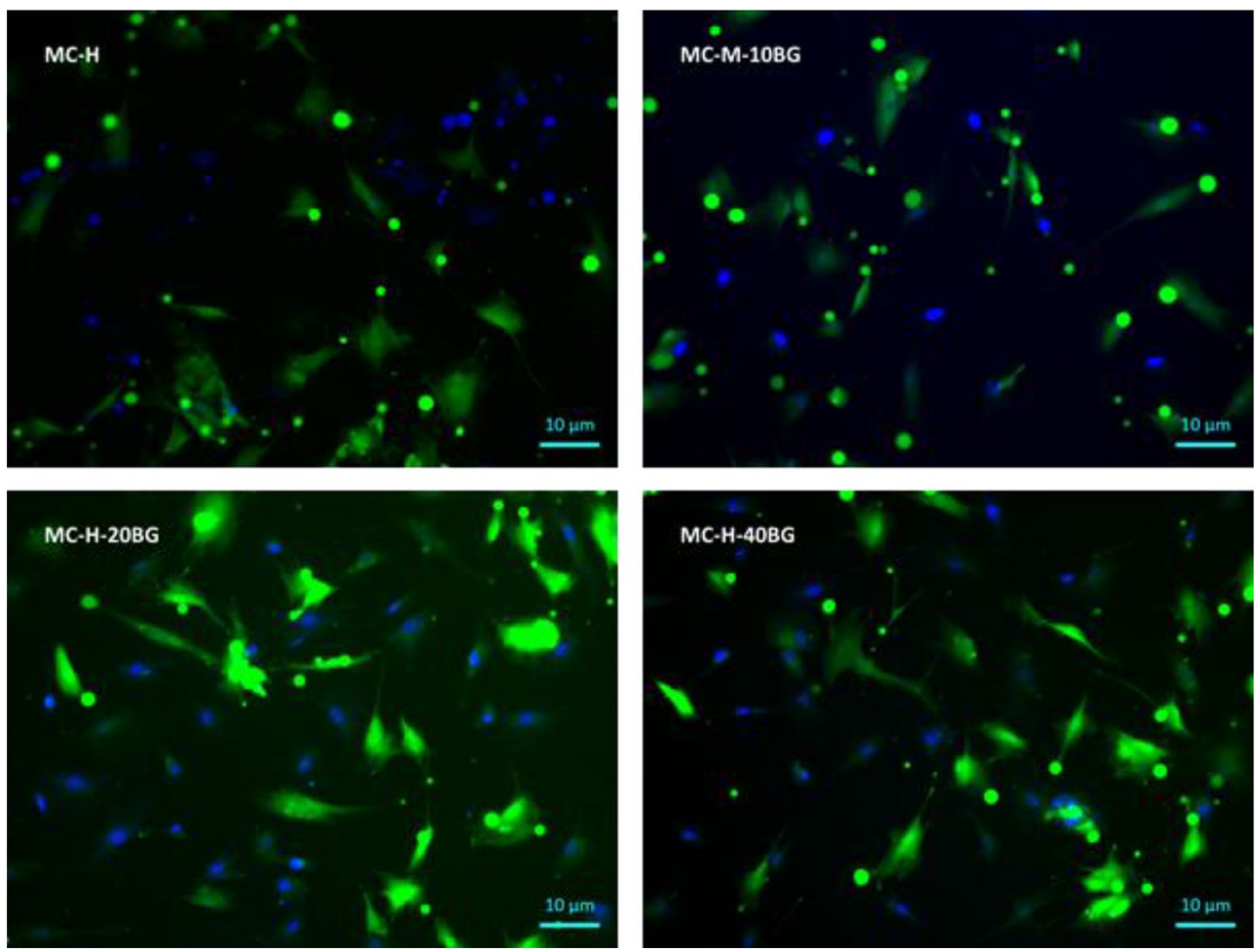

Figure 4: Fluorescence images of hDFs grown on MC-H-based scaffolds containing different amounts of borate BG particles $24 \mathrm{~h}$ after cell seeding. No differences between the tested samples could be detected.

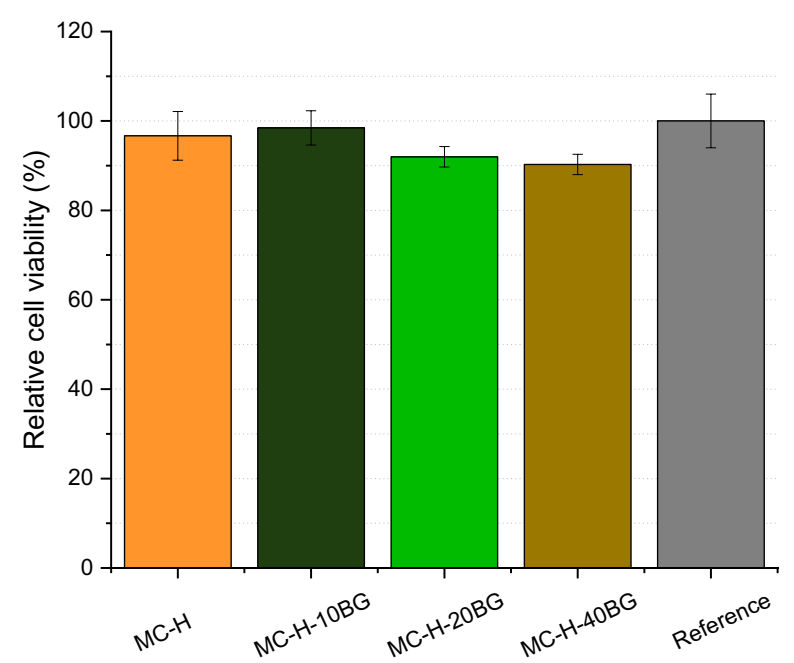

Figure 5: Relative viability of hDFs grown directly on MC-H-based scaffolds containing different amounts of BG particles after $24 \mathrm{~h}$ of culture. One-way ANOVA statistical analysis denotes no significant differences $(p<0.05)$.

time. To further improve the printability (achieving a higher output by increasing the printing speed), BG particles of borate composition were added in different amounts to the MC-H inks. As reported by Utech et al. [19], BG particles can interact with the MC-H matrix in three different ways: no clear interaction (1), specific physical interactions leading to an increase of physical cross-linking (2) or specific physical interactions leading to the disturbance of the polymeric structure (decrease of cross-linking) [19]. Our results indicate that the kind of interaction can be strongly related to the amount of BG incorporated into the MC-H matrix. In this case two of the three cross-linking possibilities reported by Utech et al. [19] seem to take place. For instance, the increase of compressive strength from low to high BG concentrations indicate an increase of cross-linking, with the exception of the ink containing $20 \mathrm{wt} \% \mathrm{BG}$. This composition seems to lead to a disturbance of the polymeric structure. Similar results have been obtained in previous studies measuring the influence of BG concentration on the compressive strength of different polymeric matrices [34-36], showing that the amount of filler (here BG particles) is crucial regarding filler-hydrogel interface reactions. In accordance to the effects found on the mechanical properties, the amount of BG particles was found to play also a crucial role on the swelling capacity of the printed scaffolds. For example, Luo et al. [35] incorporated 13-93 bioactive glass into an alginate matrix. Here the compressive strength of the composites was increased at lower BG concentrations followed by a decrease at a too high amount of incorporated BG. Killion et al. [36] reported a general increase in mechanical properties with increasing filler content. These discrepancies lead to the conclusion that different 
reinforcing mechanisms can be active when BG particles are used as filler in polymer matrices. Since the interaction of hydrogel matrices and BG particles as reactive filler depends on the particular system, and this will be determined by the quality of the filler-hydrogel interface, it is not possible to define a unique correlation between concentration, shape and (mean) size of BG particles and the mechanical properties of such composites.

Nevertheless, in general, the incorporation of BG particles leads to the generation of interfaces between the particles and the polymeric matrix, which might allow water to penetrate easier into the composite [37]. This effect could be observed for low concentrations of BG particles, where the swelling capacity was significantly higher. In samples containing $10 \mathrm{wt} \%$ of BG this seems to be the dominating factor, underlined also by the results from the contact angle measurements, which showed that the highest contact angle was measured in samples containing 10 wt $\%$ BG. With increasing BG concentrations, this effect was not observable anymore (i.e., in the $20 \mathrm{wt} \% \mathrm{BG}$ composite). A higher filler content even led to a lower swelling capacity and contact angle ( $40 \mathrm{wt} \%$ BG sample). This effect might be explained by the fact that the higher surface area due to the formed interfaces leads to a faster degradation of the polymer and especially the BG particles. It is well known that degradation rate in aqueous environments is strongly related to the surface area of materials exposed to the medium [38]. Further, as already mentioned, the three different possibilities reported by Utech et al. [19] regarding the interaction between $B G$ particles and the polymer matrix could depend on the BG content. With the incorporation of 20 $\mathrm{wt} \% \mathrm{BG}$, the dominating factor will not be the generation of interfaces due to the presence of the particles, but the interaction between BG particles and the matrix forming physical crosslinks, based on the ion-release effect from glass particles, which leads in turn to a reduced water uptake [19].

In combination, the measured mechanical properties and swelling capacities indicate that the influence of borate BG content on the $\mathrm{MC}-\mathrm{H}$ inks must be further examined in order to clearly predict the resulting properties and to choose the correct concentration of $B G$ particles leading to suitable swelling behavior and mechanical properties.

\section{Conclusion}

This study reported the great potential of using MC-H-based inks containing borate $\mathrm{BG}$ particles for $3 \mathrm{D}$ printing applications. By introducing BG particles into the MC-H-based ink, the mechanical and swelling properties could be adjusted. Different effects related to the interaction between borate BG particles and the polymer matrix are possible, depending on the concentration of the incorporated BG. However, since the interaction between filler and matrix is almost unique for each system, further tests are necessary to understand and evaluate the interface reactions between the $\mathrm{MC}-\mathrm{H}$ matrix and borate $B G$ particles. Of high interest for future studies based on these results is, therefore, the investigation of the borate BG dissolution after immersion in aqueous medium and its related ionrelease kinetics. ICP-OES measurement should be carried out to evaluate the effect of released ions on the hydrogel matrix and the possible occurrence of physical cross-linking.

Further, $\mu$-CT analysis of the 3D-printed structures should be conducted to investigate in detail the role of the filler concentration on the surrounding matrix and, therefore, on the porosity of the composite. A better understanding of the materials' porosity would give a deeper insight into the swelling behavior of the samples using different BG concentrations. Moreover, such characterization would generate knowledge on the mechanisms determining the swelling behavior. Different mechanisms, like physical and chemical cross-linking effects of the honey component and BG particles, could be acting in a time-dependent manner (weakening of the effect over time), and therefore the overall behavior of the composite hydrogels is complex and requires further in-depth studies in the future.

Cell tests suggest the biocompatibility of the newly developed composite hydrogels, indicating that the inks have potential and should be further investigated for their use in biofabrication. Here, cell studies expanding $24 \mathrm{~h}$ up to several days are of interest to further investigate the time-dependent biological performance and the stability of the generated 3D scaffolds.

\section{Materials and methods}

\section{Scaffold fabrication}

The ink was produced by heating up Dulbecco's phosphate-buffered saline (PBS, Gibco) to $50{ }^{\circ} \mathrm{C}$ and adding (under constant stirring) $10 \mathrm{wt} \%$ of MC (viscosity $4000 \mathrm{cP}$, Sigma Aldrich). After $15 \mathrm{~min}$ of stirring, $5 \mathrm{wt} \%$ of $\mathrm{H}(\mathrm{MGO}>800 \mathrm{mg} / \mathrm{kg}$, Watson \& Son, New Zealand) was added. After subsequent 15 min of stirring, different amounts of borate $\mathrm{BG}$ particles (composition in wt\%: $5.5 \mathrm{Na}_{2} \mathrm{O}, 11.1 \mathrm{~K}_{2} \mathrm{O}, 4.6 \mathrm{MgO}, 18.5 \mathrm{CaO}, 56.6 \mathrm{~B}_{2} \mathrm{O}_{3}$ and $3.7 \mathrm{P}_{2} \mathrm{O}_{5}$ [26]) were added to achieve a 10,20 or $40 \mathrm{wt} \% \mathrm{com}$ position with respect to the polymer content. The borate BG was fabricated by melt-quenching and milled to a mean particle size of 5-20 $\mu \mathrm{m}$, as described previously $[9,26]$. After another $30 \mathrm{~min}$ of stirring at $50^{\circ} \mathrm{C}$, the inks were stored in a fridge overnight in order to allow complete hydration [7]. Prior to printing, to adjust the material to room temperature, the inks were left outside the fridge for at least $1 \mathrm{~h}$. The printing process itself was performed at room temperature. The bioplotter BioScaffolder 3.1 (GeSiM mbH, Germany) was used for 3D printing experiments. This printer offers a printing head which is movable in three room axes, with a static platform and heatable cartridges, if necessary. Printing was performed using a pressure of $550 \mathrm{kPa}$ 

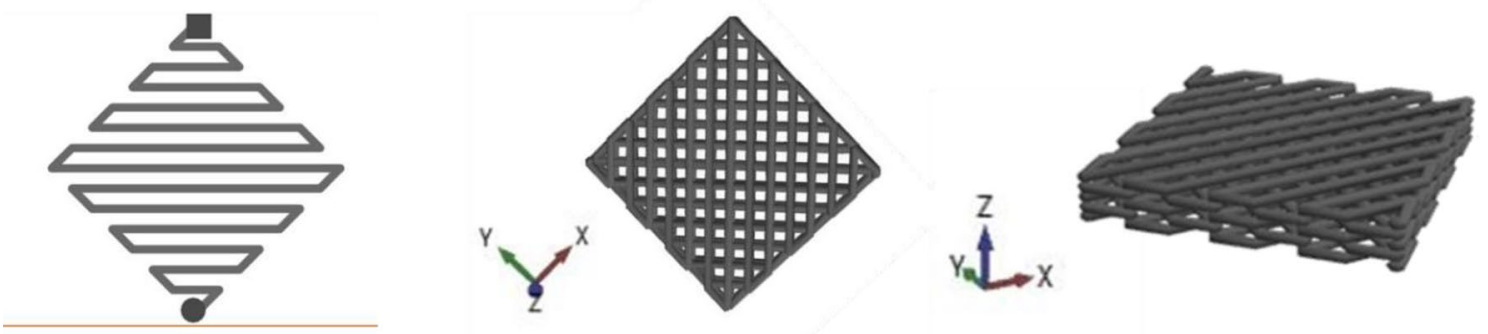

Figure 6: Schematic structure of the generated 3D scaffolds in different perspectives. Single layer with start (round) and endpoint (square) (left), top view (middle), and side view (right). All schematic structures were generated with the GeSiM printer software.

and a needle of $20 \mathrm{G}$ (inner diameter: $610 \mu \mathrm{m}$ ). The printing speed was optimized for each ink separately (MC $2.0 \mathrm{~mm} \mathrm{~s}^{-1}$, MC-H $1.6 \mathrm{~mm} \mathrm{~s}^{-1}$, MC-H-10BG $1.6 \mathrm{~mm} \mathrm{~s}^{-1}$, MC-H-20BG $2.5 \mathrm{~mm} \mathrm{~s}^{-1}$ and MC-H-40BG $4.5 \mathrm{~mm} \mathrm{~s}^{-1}$ ). Prismatic scaffolds with a height of $1.2 \mathrm{~mm}$ (4 layers, each $0.3 \mathrm{~mm}$ ), an edge length of $10 \mathrm{~mm}$ and a pore size of $0.9 \mathrm{~mm}$ were printed for each composition. The scaffold parameters had to be adjusted regarding the implemented test settings. For compression tests, scaffolds with a height of $3 \mathrm{~mm}$ (10 layers, each $0.3 \mathrm{~mm}$ ) and an edge length of $5 \mathrm{~mm}$ were generated, whereas dense scaffolds with a height of $0.6 \mathrm{~mm}$ (2 layers, each $0.3 \mathrm{~mm}$ ) and an edge length of $10 \mathrm{~mm}$ were used for contact angle measurement and preliminary cell tests.

Prior to each test, the printed 3D scaffolds were incubated at $37^{\circ} \mathrm{C}$ for $1 \mathrm{~h}$ for their stabilization [7]. The schematic structure of the generated $3 \mathrm{D}$ scaffolds in different perspectives is shown in Fig. 6, obtained by the printer software.

\section{Characterization}

Fourier transform infrared spectroscopy (FTIR, IR Affinity-1S, Shimadzu) was used to examine the chemical structure of the scaffolds. The following conditions were used: a spectral range of 4000 to $400 \mathrm{~cm}^{-1}$ and a resolution of $4 \mathrm{~cm}^{-1}$. Compression strength tests were performed using a universal testing machine (Instron 5960, USA) equipped with a load cell of 100 N. 10 replicates were tested using a cross-head speed of $10 \mathrm{~mm} / \mathrm{min}$. In addition, contact angle measurements (DSA 30, Krüss) were conducted using a $3 \mu \mathrm{l}$ water drop and at least 5 replicates of each sample type were tested. To investigate the weight change, the scaffolds were immersed in $5 \mathrm{ml}$ of Dulbecco's modified eagle medium (DMEM) for up to $72 \mathrm{~h}$. Prior to weighing, the excess of DMEM was removed, sample weights were recorded and the removed amount of DMEM was added again. The weight change was calculated by using the formula:

$$
\text { weight change }=\frac{w_{t}-w_{t 0}}{w_{0}} \times 100
$$

( $w_{0}$ is the specimen weight at time point 0 and $w_{t}$ at time $t$ ).

To evaluate the cytocompatibility of the scaffolds, a preliminary cell test using human dermal fibroblasts (hDFs) was performed [10]. Scaffolds were disinfected using UV light for $1 \mathrm{~h}$. Cells were seeded by using the technique of drop seeding to reduce the amount of cells falling from the printed structures having a slightly curved surface, to prevent cell proliferation only on the surface of the cell culture plate. For this, a $50 \mu \mathrm{l}$ drop of cell culture medium (CCM, composed of DMEM containing $10 \%$ fetal bovine serum and $1 \%$ penicillin and streptomycin) containing 100,000 hDF cells was used. After applying the DMEM drop containing the cells, samples were incubated for $15 \mathrm{~min}$ in order to let cells adhere to the surface of the samples and then filled with $1 \mathrm{ml}$ of CCM (based on a previously established protocol [10]), to cover the sample completely. After an incubation time of $24 \mathrm{~h}$ under cell culture conditions ( $95 \%$ humidity, $5 \% \mathrm{CO}_{2}$ and $37^{\circ} \mathrm{C}$ ), the viability of the cells was measured using a WST- 8 assay (Sigma Aldrich, Germany) in a dilution of $1.0 \mathrm{v} / \mathrm{v} \%$. Since the test was performed to evaluate the cytocompatibility of the scaffolds and not the proliferation of cells, the transfer of samples to a new well plate was avoided in order not to risk the destruction of the printed structure during the transfer.

In addition, the cell morphology was examined by fluorescence microscopy using two different stainings. As live staining calcein (Calcein AM, Thermo Fischer, Germany) was used, which is shown in the images in green color, and for nucleus staining 4',6-diamidino-2-phenylindole (DAPI, Thermo Fischer, Germany) (blue) was used, following the manufacturers protocols, as also discussed in the literature $[39,40]$.

\section{Funding}

Open Access funding enabled and organized by Projekt DEAL. 


\section{Data availlability}

The datasets generated during and/or analyzed during the current study are available from the corresponding author on reasonable request (A.R.B).

\section{Declarations}

Conflict of interest The authors declare no conflict of interest.

\section{Open Access}

This article is licensed under a Creative Commons Attribution 4.0 International License, which permits use, sharing, adaptation, distribution and reproduction in any medium or format, as long as you give appropriate credit to the original author(s) and the source, provide a link to the Creative Commons licence, and indicate if changes were made. The images or other third party material in this article are included in the article's Creative Commons licence, unless indicated otherwise in a credit line to the material. If material is not included in the article's Creative Commons licence and your intended use is not permitted by statutory regulation or exceeds the permitted use, you will need to obtain permission directly from the copyright holder. To view a copy of this licence, visit http://creativecommons.org/ licenses/by/4.0/.

\section{References}

1. J. Gopinathan, I. Noh, Recent trends in bioinks for 3D printing. Biomater. Res. 22(1), 11 (2018)

2. J. Li, C. Wu, P.K. Chu, M. Gelinsky, 3D printing of hydrogels: Rational design strategies and emerging biomedical applications. Mater. Sci. Eng. Res. 140, 100543 (2020)

3. E. Heymann, Studies on sol-gel transformations. I. The inverse sol-gel transformation of methylcellulose in water. Trans. Faraday Soc. 31(846), 846-864 (1935)

4. P.L. Nasatto, F. Pignon, J.L.M. Silveira, M.E.R. Duarte, M.D. Noseda, M. Rinaudo, Methylcellulose, a cellulose derivative with original physical properties and extended applications. Polymers 7(5), 777-803 (2015)

5. K. Kobayashi, C.I. Huang, T.P. Lodge, Thermoreversible gelation of aqueous methylcellulose solutions. Macromolecules 32(21), 7070-7077 (1999)

6. A. Cochis et al., 3D printing of thermo-responsive methylcellulose hydrogels for cell-sheet engineering. Materials 11(4), 1-14 (2018)

7. N. Contessi Negrini, L. Bonetti, L. Contili, S. Farè, 3D printing of methylcellulose-based hydrogels. Bioprinting 10, e00024 (2018)

8. M.K. Bain et al., Synergistic effect of salt mixture on the gelation temperature and morphology of methylcellulose hydrogel. Int. J. Biol. Macromol. 51(5), 831-836 (2012)
9. K. Schuhladen, P. Mukoo, L. Liverani, Z. Neščáková, A.R. Boccaccini, Manuka honey and bioactive glass impart methylcellulose foams antibacterial effects for wound healing applications. Biomed. Mater. 15(6), 065002 (2020)

10. K. Schuhladen, S.N. Vakamulla Raghu, L. Liverani, Z. Neščáková, A.R. Boccaccini, Production of a novel PCL-methylcellulose electrospun wound dressing by incorporating bioactive glass and Manuka honey. J. Biomed. Mater. Res. Part B

11. J. Maitra, V.K. Shukla, Cross-linking in Hydrogels - A Review. Am. J. Polym. Sci. 4, 25-31 (2014)

12. B. Hixon, Ronning-arnesen, janowiak, and sell, "investigating manuka honey antibacterial properties when incorporated into cryogel, hydrogel, and electrospun tissue engineering scaffolds." Gels 5(2), 21 (2019)

13. Y. Shan, Medicinal honey in clinical practice: viable alternative or useful adjunct in wound care management? Br. J. Nurs. 28(12), S23-S30 (2019)

14. E. Mancuso et al., Potential of Manuka Honey as a natural polyelectrolyte to develop biomimetic nanostructured meshes with antimicrobial properties. Front. Bioeng. Biotechnol. 7, 344 (2019)

15. H.W. Sung, R.N. Huang, L.H. Huang, C.C. Tsai, In vitro evaluation of cytotoxicity of a naturally occurring cross-linking reagent for biological tissue fixation. J. Biomater. Sci. Polym. Ed. 10(1), 63-78 (1999)

16. K. Schuhladen, J.A. Roether, A.R. Boccaccini, Bioactive glasses meet phytotherapeutics: the potential of natural herbal medicines to extend the functionality of bioactive glasses. Biomaterials 217, 119288 (2019)

17. L.L. Hench, The story of bioglass. J. Mater. Sci. Mater. Med. 17(11), 967-978 (2006)

18. J.R. Jones, Reprint of: review of bioactive glass: from Hench to hybrids. Acta Biomater. 23, S53-S82 (2015)

19. S. Utech, A.R. Boccaccini, A review of hydrogel-based composites for biomedical applications: enhancement of hydrogel properties by addition of rigid inorganic fillers. J. Mater. Sci. 51(1), 271-310 (2016)

20. A. Hoppe, N.S. Güldal, A.R. Boccaccini, A review of the biological response to ionic dissolution products from bioactive glasses and glass-ceramics. Biomaterials 32(11), 2757-2774 (2011)

21. V. Miguez-Pacheco, L.L. Hench, A.R. Boccaccini, Bioactive glasses beyond bone and teeth: emerging applications in contact with soft tissues. Acta Biomater. 13, 1-15 (2015)

22. S. Naseri, W.C. Lepry, S.N. Nazhat, Bioactive glasses in wound healing: hope or hype? J. Mater. Chem. B 5, 6167-6174 (2017)

23. M. Fahad, M. Gilbert, P. Dickens, Microscopy and FTIR investigations of the thermal gelation of methylcellulose in glycols. Polym. Sci. 59(1), 88-97 (2017)

24. V.M. Tul'chinsky, S.E. Zurabyan, K.A. Asankozhoev, G.A. Kogan, A.Y. Khorlin, Study of the infrared spectra of oligosaccharides in the region 1,000-40 $\mathrm{cm}^{-1}$. Carbohydr. Res. 51(1), 1-8 (1976) 
25. A. Yao, M.N. Rahaman, J. Lin, W. Huang, Structure and crystallization behavior of borate-based bioactive glass. J. Mater. Sci. 42(23), 9730-9735 (2007)

26. K. Schuhladen, X. Wang, L. Hupa, A.R. Boccaccini, Dissolution of borate and borosilicate bioactive glasses and the in $\mathrm{fl}$ uence of ion $(\mathrm{Zn}, \mathrm{Cu})$ doping in di ff erent solutions. J. Non-Cryst. Solids 502, 22-34 (2018)

27. K. Schütz, A.-M. Placht, B. Paul, S. Brüggemeier, M. Gelinsky, A. Lode, Three-dimensional plotting of a cell-laden alginate/ methylcellulose blend: towards biofabrication of tissue engineering constructs with clinically relevant dimensions. J. Tissue Eng. Regen. Med. 11(5), 1574-1587 (2017)

28. H. Li, Y.J. Tan, K.F. Leong, L. Li, 3D bioprinting of highly thixotropic alginate/methylcellulose hydrogel with strong interface bonding. ACS Appl. Mater. Interfaces 9(23), 20086-20097 (2017)

29. E. Hodder et al., Investigating the effect of sterilisation methods on the physical properties and cytocompatibility of methyl cellulose used in combination with alginate for 3D-bioplotting of chondrocytes. J. Mater. Sci. Mater. Med. 30(1), 10 (2019)

30. C.H. Chen et al., Novel living cell sheet harvest system composed of thermoreversible methylcellulose hydrogels. Biomacromol 7(3), 736-743 (2006)

31. L. Li, H. Shan, C.Y. Yue, Y.C. Lam, K.C. Tam, X. Hu, Thermally induced association and dissociation of methylcellulose in aqueous solutions. Langmuir 18(20), 7291-7298 (2002)

32. P. Zheng, L. Li, X. Hu, X. Zhao, Sol-gel transition of methylcellulose in phosphate buffer saline solutions. J. Polym. Sci. Part B 42(10), 1849-1860 (2004)
33. S. Thirumala, J. Gimble, R. Devireddy, Methylcellulose based thermally reversible hydrogel system for tissue engineering applications. Cells 2(3), 460-475 (2013)

34. K. Schuhladen, B. Lukasiewicz, P. Basnett, I. Roy, A.R. Boccaccini, Comparison of the influence of $45 \mathrm{~S} 5$ and $\mathrm{Cu}$-containing $45 \mathrm{~S} 5$ bioactive glass (BG) on the biological properties of novel polyhydroxyalkanoate (PHA)/BG composites. Materials (Basel) 13(11), 2607 (2020)

35. G. Luo et al., 13-93 bioactive glass/alginate composite scaffolds $3 \mathrm{D}$ printed under mild conditions for bone regeneration. RSC Adv. 7(20), 11880-11889 (2017)

36. J.A. Killion et al., Hydrogel/bioactive glass composites for bone regeneration applications: synthesis and characterisation. Mater. Sci. Eng. C 33(7), 4203-4212 (2013)

37. J. Jones, A. Clare, Bio-Glasses: An Introduction (Wiley, New York, 2012).

38. A.M. Deliormanli, Size-dependent degradation and bioactivity of borate bioactive glass. Ceram. Int. 39(7), 8087-8095 (2013)

39. S. Tansaz, A.K. Durmann, R. Detsch, A.R. Boccaccini, Hydrogel films and microcapsules based on soy protein isolate combined with alginate. J. Appl. Polym. Sci. 134(4), 1-9 (2017)

40. L. Liverani, M.S. Killian, A.R. Boccaccini, Fibronectin functionalized electrospun fibers by using benign solvents: best way to achieve effective functionalization. Front. Bioeng. Biotechnol. 7, $1-12(2019)$ 